\title{
Diet quality and attention capacity in European adolescents: the Healthy Lifestyle in Europe by Nutrition in Adolescence (HELENA) study
}

\author{
Pontus Henriksson $^{1 *}$, Magdalena Cuenca-García ${ }^{2,3}$, Idoia Labayen ${ }^{4}$, Irene Esteban-Cornejo ${ }^{1}$, \\ Hanna Henriksson ${ }^{1}$, Mathilde Kersting ${ }^{5}$, Jeremy Vanhelst ${ }^{6,7}$, Kurt Widhalm ${ }^{8,9}$, Frederic Gottrand ${ }^{6,7}$, \\ Luis A. Moreno ${ }^{10}$ and Francisco B. Ortega ${ }^{1,11}$ on behalf of the HELENA study group $\dagger$ \\ ${ }^{1}$ PROmoting FITness and Health through physical activity (PROFITH) Research Group, Department of Physical Education and \\ Sports, Faculty of Sport Sciences, University of Granada, Granada 18071, Spain \\ ${ }^{2}$ Department of Physical Education, School of Education, University of Cadiz, Puerto Real 11519, Spain \\ ${ }^{3}$ Department of Medical Physiology, School of Medicine, University of Granada, Granada 18016, Spain \\ ${ }^{4}$ Department of Health Sciences, Public University of Navarra, Pamplona 31008, Spain \\ ${ }^{5}$ Research Institute of Child Nutrition (FKE) Dortmund, University of Bonn, Heinstück 111, 44225 Dortmund, Germany \\ ${ }^{6}$ Inserm, LIRIC, UMR 995, Univ. Lille, CHU Lille, F-59000 Lille, France \\ ${ }^{7}$ Univ. Lille, Inserm, CHU Lille, CIC 1403 - Centre d'investigation clinique, F-59000 Lille, France \\ ${ }^{8}$ Department of Pediatrics, Paracelsus Medical University, Salzburg 5020, Austria \\ ${ }^{9}$ Department of Pediatrics, Medical University of Vienna, Vienna 1090, Austria \\ ${ }^{10}$ Growth, Exercise, Nutrition and Development (GENUD) Research Group, University of Zaragoza, Zaragoza 50009, Spain \\ ${ }^{11}$ Department of Biosciences and Nutrition, Karolinska Institute, Huddinge 14183, Sweden
}

(Submitted 12 October 2016 - Final revision received 25 April 2017 - Accepted 18 May 2017 - First published online 30 June 2017)

\begin{abstract}
Adolescence represents an important period for the development of executive functions, which are a set of important cognitive processes including attentional control. However, very little is known regarding the associations of nutrition with components of executive functions in adolescence. Thus, the aim of this study was to investigate associations of dietary patterns and macronutrient composition with attention capacity in European adolescents. This cross-sectional study included 384 ( 165 boys and 219 girls) adolescents, aged 12.5-17.5 years, from five European countries in the Healthy Lifestyle in Europe by Nutrition in Adolescence study. Attention capacity was examined using the d2 Test of Attention. Dietary intake was assessed through two non-consecutive $24 \mathrm{~h}$ recalls using a computer-based self-administered tool. Three dietary patterns (diet quality index, ideal diet score and Mediterranean diet score) and macronutrient/fibre intakes were calculated. Linear regression analysis was conducted adjusting for age, sex, BMI, maternal education, family affluence scale, study centre and energy intake (only for Mediterranean diet score). In these adjusted regression analyses, higher diet quality index for adolescents and ideal diet score were associated with a higher attention capacity (standardised $\beta=0 \cdot 16, P=0.002$ and $\beta=0 \cdot 15, P=0 \cdot 005$, respectively). Conversely, Mediterranean diet score or macronutrient/fibre intake were not associated with attention capacity $(P>0 \cdot 05)$. Our results suggest that healthier dietary patterns, as indicated by higher diet quality index and ideal diet score, were associated with attention capacity in adolescence. Intervention studies investigating a causal relationship between diet quality and attention are warranted.
\end{abstract}

Key words: Cognitive performance: Cognition: Dietary patterns: Diet quality: Executive functions

Executive functions involve a set of cognitive processes, such as attentional control, inhibitory control, working memory and cognitive flexibility, as well as reasoning, problem solving and planning. Executive functions are considered to be of great importance not only for academic performance, but also for mental and physical health as well as for overall success in life $\mathrm{e}^{(1,2)}$. Within executive functions, attentional control is a major component. Attentional control enables us to selectively attend to certain stimuli while inhibiting others and is therefore important in any learning process ${ }^{(2)}$. Adolescence represents an important period for the development of executive functions ${ }^{(3)}$, and therefore it may be important to identify modifiable factors,

Abbreviations: DASH, Dietary Approaches to Stop Hypertension; DQI-A, Diet Quality Index for adolescents; FAS, family affluence scale; HELENA, Healthy Lifestyle in Europe by Nutrition in Adolescence.

* Corresponding author: P. Henriksson, email pontus.tm.henriksson@gmail.com

$\dagger$ HELENA study group members are listed in the Supplementary material. 
such as health behaviours, which can promote cognitive development in adolescence. Diet and physical activity behaviours are two main health behaviours, and we have previously shown that higher levels of physical activity in adolescents is related to higher attention capacity ${ }^{(4)}$; however, little is known about the influence of dietary behaviours on attention capacity. A few studies have reported associations in adolescence between specific components of the diet, such as intakes of fish, vegetables, fruits and soft drinks, and cognitive or academic performance $^{(5-8)}$. However, as pointed out previously ${ }^{(9)}$, it may be difficult to separate out specific effects of single nutrients/ foods on health in epidemiological research, and thus dietary patterns may be an appealing alternative. Interestingly, a previous study showed that dietary patterns may be more strongly associated with executive functions than single nutrient/food intakes ${ }^{(10)}$ and it is possible that such findings also extend to attention capacity. Although only a few studies have examined relationships between diet and attention in childhood, several studies have investigated associations of dietary patterns and macronutrient intakes with other measures of cognitive and academic performance. For instance, healthier diets as indicated by higher scores for the Dietary Approaches to Stop Hypertension dietary pattern (DASH) ${ }^{(10)}$ and the healthy eating index ${ }^{(11)}$ have been associated with greater cognitive performance. In addition, positive associations between adherence to a Mediterranean dietary pattern and academic performance have been reported in Spanish and Greek children ${ }^{(12,13)}$. Studies have also reported positive associations of PUFA ${ }^{(14)}$ and fibre intakes ${ }^{(11)}$ with cognitive performance. Nyaradi et al. ${ }^{(15)}$ reported some evidence that a Western dietary pattern at 14 years of age was related to worse cognitive performance, including visual attention, 3 years later. However, to the best of our knowledge, no other study has explored the association of dietary patterns with attention capacity in adolescence, motivating further studies.

Data from the Healthy Lifestyle in Europe by Nutrition in Adolescence (HELENA) study provide an excellent opportunity to investigate the association of diet with attention capacity in adolescents. The HELENA study was designed to provide reliable data on nutrition and health-related variables in a relatively large sample of European adolescents and also included a measure of attention capacity in a subsample of the adolescents. Hence, we utilised data from the HELENA study to investigate the associations of dietary patterns and macronutrient composition with attention capacity in European adolescents.

\section{Methods \\ Study design and participants}

The HELENA study is a cross-sectional multicentre study, which was conducted between 2006 and 2007 in ten European cities from nine different countries ${ }^{(16-18)}$. A cluster random sample of schools was selected for each city, and detailed information about sampling procedures and study methods are provided elsewhere ${ }^{(16-19)}$. The final study sample consisted of 3528 boys and girls between 12.5 and 17.5 years of age ${ }^{(20)}$. The online
Supplementary Fig. S1 describes the sample size derivation for this study. In brief, attention capacity data were collected in six study centres ( $n$ 656) and complete data regarding attention capacity and diet were available for 400 adolescents from five study centres (Vienna in Austria, Lille in France, Dortmund in Germany, Athens in Greece and Zaragoza in Spain). In the present study, we included the 384 adolescents ( 165 boys and 219 girls) with complete data for attention, diet and basic confounders (i.e. age, sex, BMI, maternal education, family affluence scale (FAS) and study centre). Furthermore, objective physical activity data were available for 215 of the adolescents. This study was conducted according to the guidelines laid down in the Declaration of Helsinki and all procedures involving human subjects/patients were approved by the corresponding local Human Research Review Committees of the centres involved ${ }^{(21)}$. Written informed consent was obtained from all adolescents and their parents or guardians before enrolment in the study.

\section{Diet}

Dietary intake assessment. As described previously in detail $^{(19)}$, dietary intake was assessed using two nonconsecutive $24 \mathrm{~h}$ recalls, which were provided by the adolescents using a computer-based self-administered tool (the HELENA-Dietary Intake Assessment Tool) ${ }^{(22)}$. This tool was validated against $24 \mathrm{~h}$ recall interviews. In brief, average macronutrient intakes were very comparable using both methods, and there were strong correlations for all investigated nutrients ( $r$ 0.86-0.91) between the methods ${ }^{(22)}$. Trained dietitians assisted the participants if needed and the assessment was based on six meal occasions the previous day.

Rationale for the selection of dietary patterns. It was decided to examine the associations of the Diet Quality Index for adolescents (DQI-A), the ideal diet and the Mediterranean diet with attention capacity. In brief, DQI-A was chosen as this dietary index takes several important aspects of the diet into account (i.e. quality, diversity and equilibrium) and as it has previously been comprehensively validated against biomarkers, nutrients and foods ${ }^{(19)}$. Ideal diet was selected because this diet is advocated by the American Heart Association (AHA) ${ }^{(23)}$. Furthermore, the ideal diet is based on the DASH diet, which previously has been associated with cognitive performance in children ${ }^{(10)}$. Finally, the Mediterranean dietary pattern was included in the analyses because it has been consistently associated with cognition in older adults $^{(24,25)}$, and because some studies ${ }^{(12,13)}$, but not all ${ }^{(26)}$, have found a corresponding association with cognitive or academic performance in childhood.

Diet Quality Index for adolescents. A comprehensive description of the calculation of the DQI-A has been published previously $^{(19)}$. In brief, the DQI-A consisted of three components: quality, diversity and equilibrium. Daily diet was divided into nine recommended food groups: (1) water, (2) bread and cereal, (3) potatoes and grains, (4) vegetables, (5) fruits, (6) milk products, (7) cheese, (8) meat, fish and substitutes and (9) fats and oils. Dietary quality expressed whether the adolescent 
made the optimal food quality choices within a food group and was represented by a 'preference group' (i.e. the healthiest foods: cereal/brown bread, fresh fruit and fish), an 'intermediate group' (e.g. white bread, minced meat) and a 'low-nutrient, energy-dense group' (i.e. the unhealthiest foods: soft drinks, sweet snacks and chicken nuggets) using predefined criteria. The dietary quality score was then calculated by multiplying the amount (in g) of the foods consumed with a weighing factor ( +1 for preference group, 0 for intermediate group and -1 for the low-nutrient, energy-dense group) divided by the total amount of food. The diet quality score was expressed as a percentage, and thus it could vary between -100 and $100 \%$. Dietary diversity describes the degree of variation in the diet. This score was obtained by assigning 1 point for each food group that had at least one serving at the preference level, which was subsequently divided by 9 (which represents the maximum score) and then expressed as a percentage between 0 and $100 \%$. Dietary equilibrium was calculated as the difference between an adequacy score (percentage of food groups with intake above the minimum recommended intake) and the excess component (percentage of food groups exceeding the upper level of the recommended intake) and ranged between 0 and $100 \%$. DQI-A was calculated as the mean of the three components and could therefore range from -33 to $100 \%$, with higher values reflecting a higher diet quality. The DQI-A score was calculated for each day, and the average daily score was used as an indicator of the overall diet quality. The DQI-A score was also categorised in quartiles (quartile 1: $<36.7 \%$; quartile $2: 36 \cdot 7-51 \cdot 0 \%$; quartile $3: 51 \cdot 1-61 \cdot 1 \%$; and quartile 4 : $>61 \cdot 2 \%)$.

Ideal diet score. The ideal diet score, as proposed by the AHA, is based on the DASH diet ${ }^{(23)}$. It consists of five dietary indicators and cut-off values to define a healthy - that is, an ideal diet $^{(23)}$. As described previously ${ }^{(27)}$, cut-off values for ideal intake levels for a $8368 \mathrm{~kJ} / \mathrm{d}(2000 \mathrm{kcal} / \mathrm{d})$ diet were: (i) $\geq 400 \mathrm{~g}$ of fruits and vegetables, (ii) $\geq 28 \mathrm{~g}$ of fish, (iii) $\geq 28$ gramequivalent servings of fibre-rich grains $(\geq 1 \cdot 1 \mathrm{~g}$ of fibre/10 $\mathrm{g}$ of carbohydrate), (iv) $<1500 \mathrm{mg} \mathrm{Na}$ and (v) $\leq 145 \mathrm{ml}$ of soft drinks. We scaled these cut-off values individually for energy intakes other than $8368 \mathrm{~kJ}(2000 \mathrm{kcal})$. Each dietary indicator at ideal level represented 1 point and, thus the ideal diet score can range between 0 and 5 .

Mediterranean diet score for adolescents. Adherence to the Mediterranean dietary pattern was assessed using an adapted version of the traditional Mediterranean Diet Score as reported by Trichopoulou et $a l .{ }^{(28)}$. A score for adherence to the Mediterranean diet for adolescents was calculated using nine components: vegetables (excluding potatoes), fruits and nuts (including seeds and olives and excluding juices), cereals (bread and rolls, flour, cereals, rice, pasta, potatoes), legumes (excluding fresh peas, sweet maize and broad beans), fish (including seafood), the ratio of unsaturated fat:SFA, dairy products (white milk, buttermilk, yogurt, fromage blanc and cheese), meat (including poultry) and alcohol. Age- and sex-specific medians for intakes of the nine components were derived from the whole HELENA sample with complete dietary data ( $n$ 2330). Subsequently, 1 point was assigned to participants with an intake at or above the median for the seven beneficial components (i.e. vegetables, fruits and nuts, cereals, legumes, fish, the unsaturated:SFA fraction and dairy products), whereas intakes below the median were assigned 0 points. Meat and alcohol were scored in reverse as these components are presumed to be detrimental. Therefore, no intake of alcohol and a meat intake below the median were scored as 1 point each, whereas any alcohol consumption and higher intake of meat (i.e. at or above median) were assigned 0 points. Hence, the possible score for the Mediterranean diet ranged from 0 to 9 , with higher values reflecting a higher adherence to the Mediterranean diet.

Macronutrient and fibre intakes. Nutrient intakes were calculated using the German Food Code and Nutrient Data Base (Bundeslebensmittelschlüssel, version II.3.1) ${ }^{(29)}$, and the Multiple Source Method was used to estimate the usual dietary intake of nutrients and foods accounting for within-person variability $^{(30)}$.

\section{Attention capacity}

Attention capacity was assessed using the d2 Test of Attention $(\mathrm{d} 2 \mathrm{~T})^{(31,32)}$ administered in a classroom under the supervision of a HELENA fieldworker as previously described ${ }^{(4)}$. The $\mathrm{d} 2 \mathrm{~T}$ measures selective attention and response inhibition, and its reliability and validity have been tested previously in adolescents ${ }^{(32)}$. In brief, the $\mathrm{d} 2 \mathrm{~T}$ is a paper and pencil test consisting of fourteen different rows, each containing forty-seven randomly mixed letters ('p' and 'd'). The letters 'p' and 'd' appear with one or two dashes above or below each letter. Participants were instructed to mark all ' $d$ ' that appeared with two dashes (i.e. relevant elements) at a rate of $20 \mathrm{~s} /$ row. The remaining combinations of characters were considered as irrelevant elements. The complete duration of the test was $4 \mathrm{~min}$ and $40 \mathrm{~s}$ with no pauses during the test. Attention capacity was calculated as the number of correctly identified relevant elements minus commissions (i.e. number of irrelevant elements marked), and subsequently this score was transformed into age-specific percentiles. Hence, this variable ranges between 1 and 99, with higher values indicating a higher attention capacity.

\section{Covariates}

In this study, we considered age, sex, BMI, maternal education, FAS, study centre and physical activity as covariates ${ }^{(4,33)}$. Weight and height were measured using standard procedures as previously described ${ }^{(18)}$. BMI was calculated as weight (in $\mathrm{kg}$ ) divided by height (in $\mathrm{m}^{2}$ ) and classified into categories (i.e. underweight, normal weight, overweight and obese) using international cut-off values described by Cole \& Lobstein ${ }^{(34)}$. Information on maternal educational attainment and FAS, two indicators of socioeconomic status, was obtained through two questionnaires that were filled in by the mothers and adolescents, respectively. Maternal educational attainment 
was classified as elementary school, middle school, high school and university. FAS was categorised as low/medium/ high as previously described ${ }^{(35)}$. Physical activity was assessed using an accelerometer (model GT1M; ActiGraph) as described in detail previously ${ }^{(36)}$. In brief, adolescents were instructed to wear the accelerometer for 7 consecutive days during waking hours apart from time spent in water-based activities. The epoch was set at $15 \mathrm{~s}$ and moderate-to-vigorous physical activity was defined as $\geq 2000$ counts/min, as argued previously ${ }^{(37)}$. Accelerometry inclusion criteria were as follows: (i) a minimum of 3 measurement days, (ii) at least $8 \mathrm{~h} / \mathrm{d}$ of valid records and (iii) deletion of $20 \mathrm{~min}$ of consecutive epochs with 0 counts; these criteria showed the best fair trade-off between the number of days needed to identify the usual physical activity level (reliability of at least 0.80) and statistical power $^{(38)}$.

\section{Statistical analysis}

As this was a secondary analysis of HELENA data, we did not calculate statistical power a priori. To investigate linear associations between diet $(x)$ and attention capacity $(y)$, regression analyses were performed. We created three regression models: (1) unadjusted model, (2) model adjusted for basic confounders, that is, age, sex, BMI, maternal education (no university degree $v$. university degree), FAS (low/medium/high) and study centre (dummy variables) and (3) model adjusted for basic confounders in model 2 plus physical activity. All models with the Mediterranean diet were further adjusted for energy intake. We examined potential sex interactions by including an interaction term (i.e. diet variable $\times$ sex) in the adjusted regression models. None of the interaction terms was statistically significant $(P>0.05)$, and thus results are presented for boys and girls together. We conducted ANCOVA, with a Bonferroni post hoc test, to examine whether attention capacity scores differed between quartiles of DQI-A and scores of ideal diet (Fig. 1). The analyses were adjusted for basic confounders, that is, age, sex, BMI, maternal educational attainment (no university degree $v$. university degree), FAS (low/medium/ high as dummy variables) and study centre (dummy variables). Independent $t$ tests or $\chi^{2}$ tests were conducted to test differences between boys and girls. $P<0.05$ was considered statistically significant and all hypothesis tests were two-sided. Statistical analyses were performed using SPSS Statistics 22 (IBM)

Sensitivity analysis. There were no differences between the 384 adolescents in the present study and the rest of the participants in the HELENA study in terms of average age, weight, height, BMI and sex distribution $(P \geq 0 \cdot 18)$. Furthermore, there were no differences between ideal diet score $(P=0 \cdot 70)$ and Mediterranean diet score $(P=0 \cdot 17)$ between the groups, yet participants in the current study had a lower DQI-A than the rest of the participants in the HELENA study $(48 \cdot 4 v .51 \cdot 3, P=0.002)$. In addition, we re-ran all analyses after excluding underreporters who were defined as those having energy intake divided by estimated BMR $\leq 0 \cdot 96^{(19)}$. In these analyses, results were virtually the same (data not shown).

\section{Results}

\section{Descriptive statistics}

Descriptive data of the adolescents are presented in Table 1. Overall, $22.7 \%$ of the adolescents were overweight or obese. A higher proportion of the boys met the physical activity recommendations in comparison with girls $(P<0 \cdot 001)$. In contrast, girls had on average a higher DQI-A and ideal diet score than boys $(P \leq 0 \cdot 001)$, whereas no differences were observed between the sexes for attention capacity. All dietary patterns were correlated with each other, that is, DQI-A $v$. ideal diet score $(r 0.47, P<0.001)$, DQI-A $v$. Mediterranean diet score $(r 0.35, P<0.001)$ and ideal diet score $v$. Mediterranean diet score $(r 0 \cdot 27, P<0 \cdot 001)$. Furthermore, there was no significant difference in the Mediterranean diet score between adolescents from the centre-north of Europe (Vienna, Lille and Dortmund) and those from south Europe (Athens and Zaragoza) (4.21 $v$. 4.56, $P=0.063)$.

\section{Dietary patterns and macronutrients v. attention capacity}

Table 2 presents associations of dietary patterns and diet composition with attention capacity. Overall, there was little evidence of associations of single macronutrient and fibre intakes with attention capacity. On the other hand, DQI-A and ideal diet score were positively associated with attention capacity in unadjusted analyses $(P \leq 0 \cdot 019)$ and these associations remained statistically significant after adjustments for age, sex, BMI, maternal education attainment, FAS and study centre $(P \leq 0.005)$. Thus, in adjusted analyses, each standard deviation increase in DQI-A and ideal diet score was associated with 0.16 and $0 \cdot 15 \mathrm{SD}$ greater attention capacity, respectively. After further adjustments for time spent in moderate-to-vigorous physical activity per day, the strength of the associations of DQI-A and ideal diet score with attention capacity were very comparable. However, the association of ideal diet with attention capacity did not quite reach statistical significance $(P=0.054)$ due to the fact that objective physical activity data were only available for $56 \%$ of the participants. Thus, the sample size was reduced from 384 to 215 when physical activity was included in the models. In contrast, adherence to the Mediterranean diet was not associated with attention capacity (Table 2). Fig. 1 shows the differences in attention capacity according to the quartiles of the DQI-A (a) and points of the ideal diet score (b) after adjustments for basic confounders. Average attention capacity in the highest quartile of the DQI-A was significantly higher than the average attention capacity in the lowest $(54.2 v .41 \cdot 0, P=0.009)$.

\section{Components of dietary patterns v. attention capacity}

The online Supplementary Table S1 presents the association of the components of the three dietary patterns with attention capacity. In brief, a higher diet quality and diet equilibrium components of the DQI-A were associated with higher attention capacity. Furthermore, 1 point from the soft drink and $\mathrm{Na}$ consumption components (i.e. lower consumptions) from the ideal diet score was associated with higher attention capacity compared with 0 points (i.e. higher consumption) in adjusted analyses. 
Table 1. Descriptive characteristics of European adolescents from the Healthy Lifestyle in Europe by Nutrition in Adolescence study (Mean values and standard deviations; percentages)

\begin{tabular}{|c|c|c|c|c|c|c|c|}
\hline & \multicolumn{2}{|c|}{ All $(n$ 384) } & \multicolumn{2}{|c|}{ Boys ( $n$ 165) } & \multicolumn{2}{|c|}{ Girls (n 219) } & \multirow[b]{2}{*}{$P_{\text {for sex }}$} \\
\hline & Mean & SD & Mean & SD & Mean & SD & \\
\hline \multicolumn{8}{|l|}{ Physical characteristics } \\
\hline Age (years) & $14 \cdot 7$ & $1 \cdot 3$ & $14 \cdot 8$ & $1 \cdot 3$ & 14.6 & $1 \cdot 2$ & 0.059 \\
\hline Weight (kg) & 59.5 & $13 \cdot 7$ & 64.5 & $16 \cdot 4$ & $55 \cdot 8$ & $9 \cdot 7$ & $<0.001$ \\
\hline Height (cm) & $165 \cdot 8$ & $9 \cdot 1$ & $170 \cdot 6$ & $9 \cdot 7$ & $162 \cdot 3$ & $6 \cdot 6$ & $<0.001$ \\
\hline BMI $\left(\mathrm{kg} / \mathrm{m}^{2}\right)$ & 21.5 & 3.6 & 21.9 & $4 \cdot 1$ & 21.1 & 3.2 & 0.032 \\
\hline Overweight/obese (\%)† & \multicolumn{2}{|c|}{$22 \cdot 7$} & \multicolumn{2}{|c|}{29.7} & \multicolumn{2}{|c|}{17.4} & 0.004 \\
\hline Moderate-to-vigorous PA (min/d)‡ & $59 \cdot 2$ & 23.4 & 71.5 & $25 \cdot 2$ & $50 \cdot 3$ & $17 \cdot 6$ & $<0.001$ \\
\hline Meeting PA recommendations $(\%) \ddagger \S$ & \multicolumn{2}{|c|}{$42 \cdot 3$} & \multicolumn{2}{|c|}{$66 \cdot 3$} & \multicolumn{2}{|c|}{$25 \cdot 4$} & $<0.001$ \\
\hline \multicolumn{8}{|l|}{ Socioeconomic status } \\
\hline FAS (\%) & \multirow{2}{*}{\multicolumn{2}{|c|}{$7 \cdot 3$}} & \multirow{2}{*}{\multicolumn{2}{|c|}{$4 \cdot 8$}} & & & 0.26 \\
\hline Low & & & & & \multicolumn{2}{|c|}{$9 \cdot 1$} & \\
\hline Medium & \multicolumn{2}{|c|}{$58 \cdot 1$} & \multicolumn{2}{|c|}{$60 \cdot 6$} & \multicolumn{2}{|c|}{$56 \cdot 2$} & \\
\hline High & \multicolumn{2}{|c|}{$34 \cdot 6$} & \multicolumn{2}{|c|}{34.5} & \multicolumn{2}{|c|}{34.7} & \\
\hline Maternal educational attainment (\%) & & & & & & & 0.57 \\
\hline Primary and secondary education & \multicolumn{2}{|c|}{$76 \cdot 6$} & \multicolumn{2}{|c|}{$75 \cdot 2$} & \multicolumn{2}{|c|}{$77 \cdot 6$} & \\
\hline University degree & \multicolumn{2}{|c|}{$23 \cdot 4$} & \multicolumn{2}{|c|}{$24 \cdot 8$} & \multicolumn{2}{|c|}{$22 \cdot 4$} & \\
\hline \multicolumn{8}{|l|}{ Dietary characteristics } \\
\hline DQI-A (-33-100\%) & $48 \cdot 4$ & $16 \cdot 7$ & $45 \cdot 0$ & $17 \cdot 8$ & $50 \cdot 9$ & $15 \cdot 5$ & 0.001 \\
\hline Ideal diet score $(0-5)$ & 1.07 & 1.07 & 0.78 & 0.88 & 1.29 & $1 \cdot 16$ & $<0.001$ \\
\hline Mediterranean diet score (0-9) & 4.29 & 1.58 & 4.18 & 1.56 & 4.38 & 1.59 & 0.23 \\
\hline Energy $(\mathrm{kJ} / \mathrm{d})$ & 8715 & 3054 & 10021 & 3289 & 7732 & 2448 & $<0.001$ \\
\hline Energy (kcal/d) & 2083 & 730 & 2395 & 786 & 1848 & 585 & $<0.001$ \\
\hline Carbohydrates (\% of energy) & $50 \cdot 7$ & $7 \cdot 7$ & 50.5 & $7 \cdot 7$ & $50 \cdot 8$ & 7.6 & 0.72 \\
\hline Protein (\% of energy) & $15 \cdot 6$ & 3.5 & $16 \cdot 0$ & 3.5 & $15 \cdot 3$ & $3 \cdot 1$ & 0.049 \\
\hline Total fat (\% of energy) & $32 \cdot 6$ & $6 \cdot 3$ & $31 \cdot 8$ & 6.5 & $33 \cdot 2$ & $6 \cdot 1$ & 0.036 \\
\hline SFA (\% of energy) & 13.5 & $2 \cdot 8$ & $13 \cdot 1$ & $2 \cdot 9$ & 13.9 & $2 \cdot 6$ & 0.006 \\
\hline MUFA (\% of energy) & $12 \cdot 1$ & 3.0 & 11.9 & 3.0 & $12 \cdot 1$ & $2 \cdot 9$ & 0.51 \\
\hline PUFA (\% of energy) & 4.5 & 1.4 & $4 \cdot 3$ & 1.4 & 4.7 & 1.4 & 0.002 \\
\hline Total fibre $(g / d)$ & $18 \cdot 6$ & 7.3 & 19.5 & 7.7 & 17.9 & $6 \cdot 8$ & 0.027 \\
\hline Soluble fibre $(g / d)$ & $5 \cdot 8$ & $2 \cdot 1$ & $6 \cdot 2$ & $2 \cdot 3$ & 5.6 & $2 \cdot 0$ & 0.004 \\
\hline Insoluble fibre $(\mathrm{g} / \mathrm{d})$ & 13.4 & 5.4 & $14 \cdot 0$ & $5 \cdot 7$ & $12 \cdot 9$ & $5 \cdot 1$ & 0.051 \\
\hline Attention capacity percentile score (1-99) & $47 \cdot 2$ & $27 \cdot 9$ & 47.4 & $28 \cdot 1$ & $47 \cdot 0$ & 27.7 & 0.91 \\
\hline
\end{tabular}

PA, physical activity; FAS, family affluence scale; DQI-A, Diet Quality Index for adolescents.

* $P$ value of an independent test (continuous variables) or $X^{2}$ test (categorical variables) between boys and girls. † Overweight and obesity according to Cole \& Lobstein ${ }^{(34)}$.

$\ddagger n 215$ (eighty-nine boys and 126 girls).

$\S \geq 60$ min moderate-to-vigorous PA per day $^{(39)}$.

\section{Discussion}

The main finding of this study was that a healthier dietary pattern, as indicated by higher DQI-A and ideal diet score, was associated with greater attention capacity in European adolescents. Importantly, these associations were largely unaffected by adjustment for relevant confounders such as age, sex, BMI, socioeconomic status and physical activity. Our results may be of importance as they suggest that attention capacity, an important component in executive functions, is influenced by healthy dietary patterns in adolescence.

One interesting finding is that we observed consistent associations of DQI-A and ideal diet score with attention capacity, but there was little evidence for associations of separate dietary pattern components or macronutrient composition with attention capacity. More specifically, although the diet quality and diet equilibrium components of the DQI-A were related to attention capacity, soft drink and $\mathrm{Na}$ consumption (from the ideal diet score) were the only food items that were associated with attention capacity in adjusted analyses. The dietary patterns examined in this study also included several potentially important food items such as fish, vegetables and fruits, which previously have been linked to cognitive performance or academic achievement in youth ${ }^{(5-8)}$. However, in this study, these food items were not consistently associated with attention capacity. Hence, our finding suggests that examining the diet as a whole - that is, in terms of dietary patterns - is a stronger determinant of attention capacity than examining single nutrients/foods, agreeing with a previous study on diet and cognitive performance in children ${ }^{(10)}$. There are several potential reasons why dietary patterns may be more strongly related to cognitive performance than single nutrients/foods. First, the effect of single nutrients/foods on health may be too small to detect, whereas the combined effect of several nutrients/foods may be large enough to be detectable ${ }^{(9)}$. In our adjusted analysis with the components of ideal diet score, only two of the five components (soft drinks and $\mathrm{Na}$ ) were significantly associated with attention capacity, whereas two other components (fruit/vegetables and fish) had estimates in the expected direction, but did not quite reach statistical significance. Nevertheless, the combined effect of the variables in the ideal diet score exhibited a strong association with attention capacity 
consistent across the regression models. Second, variables related to the overall diet, such as macronutrient intakes, may be measured more precisely than single micronutrients ${ }^{(40)}$, which may also be the case for dietary patterns. Finally, as pointed out previously ${ }^{(9,41)}$, nutrients/food in a dietary pattern may also act in a synergistic manner to influence health. Hence, dietary patterns may offer a more holistic description of dietary habits than single nutrients/foods.

Considering that the diet as a whole, rather than single items, was of importance for attention capacity, potential mechanisms behind our findings are difficult to elucidate. Nevertheless, Western diets (which are poor in vegetables, fruits and fish, but rich in meat and refined sugars) have been linked to impaired cognitive function, and suggested mechanisms underlying these associations include increased oxidative stress, neuroinflammation and impaired blood-brain barrier integrity ${ }^{(42)}$. Furthermore, vegetables and fruits are rich in polyphenols, which may have neuroprotective properties ${ }^{(43)}$, and in micronutrients such as folate, which may be important for cognitive development ${ }^{(41)}$. Nutrients may also act in an interactive manner to influence cognitive development ${ }^{(41)}$. Another potential mechanism by which dietary factors may influence cognition is through the brain-derived neurotrophic factor, which is susceptible to changes in the diet and has an important function in the growth and survival of many types of neurons and may influence synaptic plasticity and cognitive function ${ }^{(24,44)}$. Furthermore, both insulin resistance and high blood pressure have been suggested to negatively affect cognition ${ }^{(24)}$. Hence, our identified associations of a high soft drink and $\mathrm{Na}$ consumption with worse attention capacity may be partly explained by the fact that excessive soft drink and $\mathrm{Na}$ intakes are related to the metabolic syndrome ${ }^{(45)}$ and higher blood pressure $^{(46)}$, respectively. Furthermore, high soft drink and $\mathrm{Na}$ intake are also closely connected to a Western dietary pattern, which previously has been associated with worse cognitive and academic performance in youth ${ }^{(15,47)}$.

Generally, comparisons of our results with other studies are complicated because of differences in age groups, cognitive performance tests, methods of obtaining dietary data and because dietary patterns have been derived differently (i.e. dietary patterns derived a priori $v$. factor analysis). Nevertheless, Nyaradi et al ${ }^{(15)}$ reported some evidence that a higher adherence to the Western dietary pattern at 14 years of age was associated with lower cognitive performance in tests describing visual attention, memory and attention, psychomotor functioning as well as memory and executive functions at 17 years of age. Conversely, associations with the healthy dietary pattern were less pronounced. Furthermore, Sörensen et al. ${ }^{(48)}$ conducted a randomised controlled trial that compared the effect of a healthy school lunch with the usual packed lunch from home (normally rye bread sandwiches). The healthy school lunch improved reading performance; however, no effect was observed on the attention capacity, which is in contrast to our findings. There are some possible explanations for the differences between the studies. Although the intervention increased the overall diet quality by increasing intakes of vegetables and fish as well as some micronutrients, this increase may have been too small to affect attention capacity. Furthermore, the 
Diet and attention capacity in adolescence

(a)

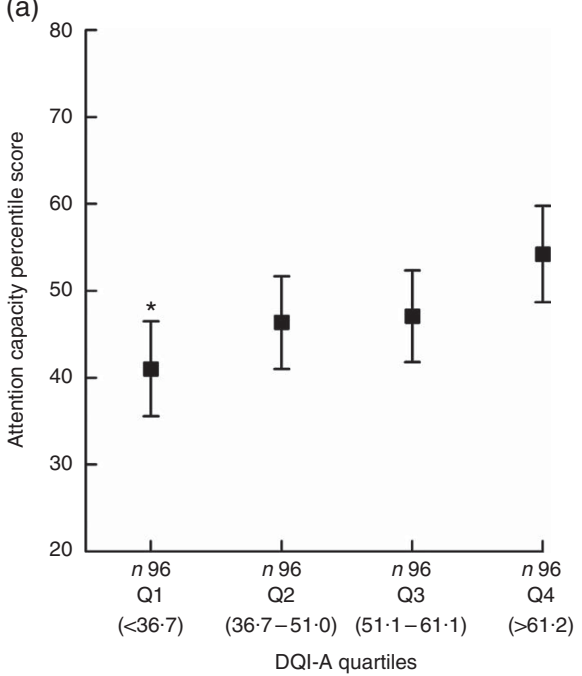

(b)

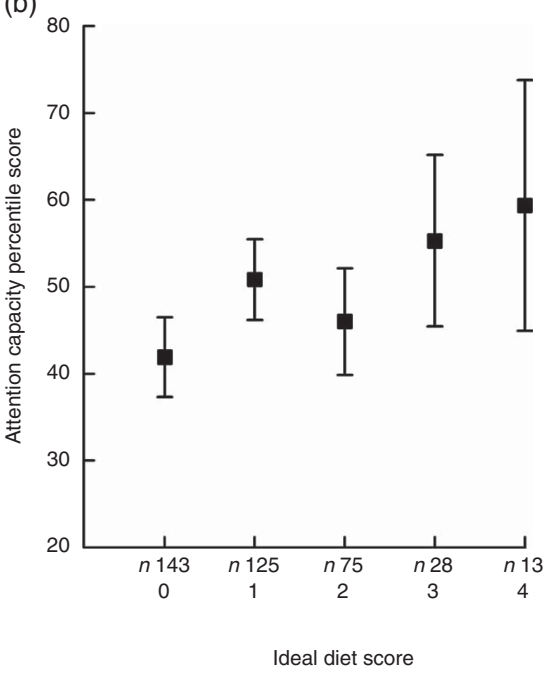

Fig. 1. Differences in attention capacity score according to (a) Diet Quality Index for adolescents (DQI-A) and (b) ideal diet score in European adolescents using ANCOVA adjusting for age, sex, BMI, maternal educational attainment, family affluence scale and study centre. Estimated marginal means with their $95 \% \mathrm{Cl}$ are presented. * Difference between quartiles $(\mathrm{Q}) 1$ and 4 in DQI-A was statistically significant $(P=0.009)$.

intervention was only performed for 3 months, which may have been too short a time period to have an effect on attention capacity. Finally, the different study designs may also be a contributing factor to the contrasting results. Indeed, the crosssectional design of the current study does not allow the possibility of drawing any conclusions regarding the direction of the relationship between diet and attention. It has been hypothesised that better executive functions enable healthier food choices $^{(49)}$ and Allan et al. ${ }^{(50)}$ suggested that the association between healthy food intake and executive functions is bidirectional. Hence, further studies are needed to elucidate the association between nutrition and cognitive performance/ executive functions.

Our results may be also reconciled to studies that have investigated associations of dietary patterns with other dimensions of cognitive performance than attention capacity in youth. For instance, Haapala et al. ${ }^{(10)}$ reported that a higher Baltic Sea diet score and DASH score in boys was associated with higher performance in Raven's coloured Progressive Matrices, which is suggested to reflect all core components of executive functions. The latter finding can be reconciled with our finding of a positive association between the ideal diet score (which is based on DASH) and attention capacity, although we did not find any evidence for a sex interaction. Furthermore, Khan et $a l .{ }^{(11)}$ reported that a higher diet quality, as indicated by the Healthy-Eating Index-2005, was associated with greater cognitive flexibility, which may be compared with our identified association between DQI-A and attention capacity. Finally, in a study of Greek preschoolers, the derived 'snacky' dietary pattern was associated with worse verbal ability, general cognitive ability and cognitive functions of the posterior cortex ${ }^{(26)}$. However, no association was identified using a 'Mediterraneanlike dietary pattern', which agrees with the results from the current study. These findings are somewhat unexpected as the Mediterranean diet has consistently been associated with higher cognitive function and reduced risk for mild cognitive impairment or Alzheimer's disease in adults ${ }^{(24,25)}$. Our results are also in contrast with two previous studies that reported positive associations of the Mediterranean diet with academic performance in youth ${ }^{(12,13)}$. One reason for this discrepancy could be that academic and cognitive performance are different concepts that are rather weakly correlated to each other and also depend on the dimension of academic and cognitive performance being examined ${ }^{(51)}$. Furthermore, diet in the studies by Esteban-Cornejo et al. and Vassiloudis et al. was measured using the KIDMED questionnaire ${ }^{(52)}$, which enquires only about some aspects of the diet (breakfast skipping, intakes of food and sweets), which are not included in the original Mediterranean diet score devised by Trichopoulou et al. ${ }^{(28)}$. Nevertheless, our supplementary analysis (online Supplementary Table S1) found no strong evidence that any of the components in our Mediterranean diet score were associated with attention capacity. Comparisons with other reports of Mediterranean diet and cognition are complicated by the fact that most studies have investigated middle-aged and older adults ${ }^{(24)}$ Thus, further studies are needed to clarify the role of the Mediterranean diet in cognition in youth.

A study by Khan et al. ${ }^{(11)}$ reported an association of higher dietary fibre intake with greater attentional inhibition. However, we were unable to replicate this finding even though our sample size was larger (384v sixty-five participants). Potential reasons for the discrepancy between the studies may be the age differences between the subjects and differences in the cognitive tests performed. Finally, we observed no evidence of an association of intakes of PUFA with attention capacity, which is partly in disagreement with the large ( $n$ 3666) study by Zhang et al. ${ }^{(14)}$ who found a positive association between PUFA intake and performance in one of the four examined cognitive tests (i.e. digit span test). Furthermore, Johnson et al. ${ }^{(53)}$ reported that an intervention increasing the intake of PUFA had a positive effect on reading ability, which was more pronounced in children with attentional problems. 
Limitations of this study include the cross-sectional design, which limits the ability to draw conclusions about causality as well as the direction of the associations. Another limitation is that we assessed one dimension of executive functions, attention capacity, using one cognitive test. Further studies are needed to examine other dimensions of executive functions than attention capacity, and could also involve more sophisticated techniques such as electroencephalography and functional MRI. Finally, we cannot rule out the fact that our results are due to the inability to adjust for all confounders. Nevertheless, we were able to adjust for several relevant confounders ${ }^{(4,33)}$ and the results were materially unchanged. Strengths of this study include the fact that data were collected in a diverse European population using carefully standardised procedures ${ }^{(16,18)}$. Another strength was that we utilised several dietary patterns.

Overall, our findings suggest that a higher DQI-A and ideal diet score are related to a higher attention capacity, which may be of importance as attentional control represents a key component in executive functions. Given that execution functions are essential for academic achievement and overall success in life ${ }^{(2)}$, our findings may be of great importance. As $1 \mathrm{sD}$ higher DQI-A and ideal diet score were associated with 0.16 and $0 \cdot 15 \mathrm{SD}$ higher attention capacity, we believe that these associations are strong enough to be relevant from a public health perspective. However, further research is needed to clarify the role of nutrition in cognitive development, including further longitudinal studies and/or randomised controlled trials.

\section{Acknowledgements}

The authors thank the adolescents who participated in the study as well as their parents and teachers. The authors also thank the fieldwork members for their efforts.

The HELENA project was supported by the European Community Sixth RTD Framework Programme (contract FOODCT-2005-007034). P. H. was supported by a grant from Henning and Johan Throne-Holst Foundation. F. B. O. and I. E.-C. were supported by the Spanish Ministry of Science and Innovation (RYC-2011-09011 and FJCI-2014-19563). H. H. was supported by grants from the Swedish Society of Medicine and the County Council of Östergötland, Sweden. In addition, this study took place under the umbrella of the ActiveBrains project, funded by the Spanish Ministry of Economy and Competitiveness (Ref. DEP2013-47540); it was further supported by the SAMID III network, RETICS, funded by the PN I+D + I 2017-2021 (Spain), ISCIII-Sub-Directorate General for Research Assessment and Promotion and the European Regional Development Fund (Ref. RD16/0022) and by the University of Granada, Plan Propio de Investigación 2016, Excellence actions: Units of Excellence, Unit of Excellence on Exercise and Health. The funders of the HELENA study had no role in the design, analysis or writing of this article. The content of this paper reflects the authors' views alone, and the European Community is not liable for any use that may be made of the information contained herein.

Author responsibilities were as follows: K. W., F. G., L. A. M. and F. B. O. designed the research; M. C.-G., J. V., K. W., F. G., L. A. M. and F. B. O. conducted the research; P. H. and F. B. O. analysed the data and had primary responsibility for the final content; P. H., M. C.-G., I. L., I. E.-C., H. H. and F. B. O. wrote the paper. All authors read and approved the final manuscript. None of the authors has any conflicts of interest to declare.

\section{Supplementary material}

For supplementary material/s referred to in this article, please visit https://doi.org/10.1017/S0007114517001441

\section{References}

1. Cukierman-Yaffe T, Kasher-Meron M, Fruchter E, et al. (2015) Cognitive performance at late adolescence and the risk for impaired fasting glucose among young adults. $J$ Clin Endocrinol Metab 100, 4409-4416.

2. Diamond A (2013) Executive functions. Annu Rev Psychol 64, 135-168.

3. Luna B (2009) Developmental changes in cognitive control through adolescence. Adv Child Dev Behav 37, 233-278.

4. Vanhelst J, Beghin L, Duhamel A, et al. (2016) Physical activity is associated with attention capacity in adolescents. J Pediatr 168, 126-131.e122.

5. Aberg MA, Aberg N, Brisman J, et al. (2009) Fish intake of Swedish male adolescents is a predictor of cognitive performance. Acta Paediatr 98, 555-560.

6. Kim SY, Sim S, Park B, et al. (2016) Dietary habits are associated with school performance in adolescents. Medicine (Baltimore) 95, e3096.

7. Sigfusdottir ID, Kristjansson AL \& Allegrante JP (2007) Health behaviour and academic achievement in Icelandic school children. Health Educ Res 22, 70-80.

8. Stea TH \& Torstveit MK (2014) Association of lifestyle habits and academic achievement in Norwegian adolescents: a cross-sectional study. BMC Public Health 14, 829.

9. Hu FB (2002) Dietary pattern analysis: a new direction in nutritional epidemiology. Curr Opin Lipidol 13, 3-9.

10. Haapala EA, Eloranta AM, Venalainen T, et al. (2015) Associations of diet quality with cognition in children - the Physical Activity and Nutrition in Children Study. Br J Nutr 114, 1080-1087.

11. Khan NA, Raine LB, Drollette ES, et al. (2015) Dietary fiber is positively associated with cognitive control among prepubertal children. J Nutr 145, 143-149.

12. Esteban-Cornejo I, Izquierdo-Gomez R, Gomez-Martinez S, et al. (2015) Adherence to the Mediterranean diet and academic performance in youth: the UP\&DOWN study. Eur $J$ Nutr 55, 1133-1140.

13. Vassiloudis I, Yiannakouris N, Panagiotakos DB, et al. (2014) Academic performance in relation to adherence to the Mediterranean diet and energy balance behaviors in Greek primary schoolchildren. J Nutr Educ Behav 46, 164-170.

14. Zhang J, Hebert JR \& Muldoon MF (2005) Dietary fat intake is associated with psychosocial and cognitive functioning of schoolaged children in the United States. J Nutr 135, 1967-1973.

15. Nyaradi A, Foster JK, Hickling S, et al. (2014) Prospective associations between dietary patterns and cognitive performance during adolescence. J Child Psychol Psychiatry 55 , $1017-1024$

16. Moreno LA, De Henauw S, Gonzalez-Gross M, et al. (2008) Design and implementation of the Healthy Lifestyle in Europe by Nutrition in Adolescence Cross-Sectional Study. Int J Obes (Lond) 32, Suppl. 5, S4-S11.

17. Moreno LA, Gonzalez-Gross M, Kersting M, et al. (2008) Assessing, understanding and modifying nutritional status, eating habits and physical activity in European 
adolescents: the HELENA (Healthy Lifestyle in Europe by Nutrition in Adolescence) Study. Public Health Nutr 11, 288-299.

18. Nagy E, Vicente-Rodriguez G, Manios Y, et al. (2008) Harmonization process and reliability assessment of anthropometric measurements in a multicenter study in adolescents. Int J Obes (Lond) 32, Suppl. 5, S58-S65.

19. Vyncke K, Cruz Fernandez E, Fajo-Pascual M, et al. (2013) Validation of the Diet Quality Index for Adolescents by comparison with biomarkers, nutrient and food intakes: the HELENA study. Br J Nutr 109, 2067-2078.

20. Beghin L, Huybrechts I, Vicente-Rodriguez G, et al. (2012) Main characteristics and participation rate of European adolescents included in the HELENA study. Arch Public Health 70, 14 .

21. Beghin L, Castera M, Manios Y, et al. (2008) Quality assurance of ethical issues and regulatory aspects relating to good clinical practices in the HELENA Cross-Sectional Study. Int $J$ Obes (Lond) 32, Suppl. 5, S12-S18.

22. Vereecken CA, Covents M, Sichert-Hellert W, et al. (2008) Development and evaluation of a self-administered computerized 24-h dietary recall method for adolescents in Europe. Int J Obes (Lond) 32, Suppl. 5, S26-S34.

23. Lloyd-Jones DM, Hong Y, Labarthe D, et al. (2010) Defining and setting national goals for cardiovascular health promotion and disease reduction: the American Heart Association's strategic Impact Goal through 2020 and beyond. Circulation 121, 586-613.

24. Hardman RJ, Kennedy G, Macpherson H, et al. (2016) Adherence to a Mediterranean-style diet and effects on cognition in adults: a qualitative evaluation and systematic review of longitudinal and prospective trials. Front Nutr 3, 22.

25. Singh B, Parsaik AK, Mielke MM, et al. (2014) Association of Mediterranean diet with mild cognitive impairment and Alzheimer's disease: a systematic review and meta-analysis. J Alzheimers Dis 39, 271-282.

26. Leventakou V, Roumeliotaki T, Sarri K, et al. (2016) Dietary patterns in early childhood and child cognitive and psychomotor development: the Rhea mother-child cohort study in Crete. Br J Nutr 115, 1431-1437.

27. Ruiz JR, Huybrechts I, Cuenca-Garcia M, et al. (2015) Cardiorespiratory fitness and ideal cardiovascular health in European adolescents. Heart 101, 766-773.

28. Trichopoulou A, Costacou T, Bamia C, et al. (2003) Adherence to a Mediterranean diet and survival in a Greek population. $N$ Engl J Med 348, 2599-2608.

29. Dehne LI, Klemm C, Henseler G, et al. (1999) The German Food Code and Nutrient Data Base (BLS II.2). Eur J Epidemiol 15, 355-359.

30. Harttig U, Haubrock J, Knuppel S, et al. (2011) The MSM program: web-based statistics package for estimating usual dietary intake using the Multiple Source Method. Eur J Clin Nutr 65, Suppl. 1, S87-S91.

31. Bates ME \& Lemay EP Jr (2004) The d2 Test of attention: construct validity and extensions in scoring techniques. $J$ Int Neuropsychol Soc 10, 392-400.

32. Brickenkamp R \& Zillmer E (1998) The d2 Test of Attention. 1st US ed. Seattle, WA: Hogrefe \& Huber Publishers.

33. Cadenas-Sanchez C, Vanhelst J, Ruiz JR, et al. (2017) Fitness and fatness in relation with attention capacity in European adolescents: The HELENA study. J Sci Med Sport 20, 373-379.

34. Cole TJ \& Lobstein T (2012) Extended international (IOTF) body mass index cut-offs for thinness, overweight and obesity. Pediatr Obes 7, 284-294

35. Iglesia I, Mouratidou T, Gonzalez-Gross M, et al. (2014) Socioeconomic factors are associated with folate and vitamin $B_{12}$ intakes and related biomarkers concentrations in European adolescents: the Healthy Lifestyle in Europe by Nutrition in Adolescence study. Nutr Res 34, 199-209.

36. Ruiz JR, Ortega FB, Martinez-Gomez D, et al. (2011) Objectively measured physical activity and sedentary time in European adolescents: the HELENA study. Am J Epidemiol 174, 173-184.

37. Guinhouya BC, Hubert H \& Zitouni D (2011) Need for unbiased computation of the moderate-intensity physical activity of youth in epidemiologic studies. Am I Prev Med $\mathbf{4 1}$, e1-e2; author reply e2-e3.

38. Martinez-Gomez D, Ruiz JR, Ortega FB, et al. (2010) Recommended levels and intensities of physical activity to avoid lowcardiorespiratory fitness in European adolescents: the HELENA study. Am J Hum Biol 22, 750-756.

39. US Department of Health and Human Services \& Physical Activity Guidelines Advisory Committee (2008) Physical activity guidelines for Americans. http://www.health.gov/ PAguidelines (accessed October 2016).

40. Nelson M, Black AE, Morris JA, et al. (1989) Between- and within-subject variation in nutrient intake from infancy to old age: estimating the number of days required to rank dietary intakes with desired precision. Am J Clin Nutr 50, 155-167.

41. Nyaradi A, Li J, Hickling S, et al. (2013) The role of nutrition in children's neurocognitive development, from pregnancy through childhood. Front Hum Neurosci 7, 97.

42. Francis H \& Stevenson R (2013) The longer-term impacts of Western diet on human cognition and the brain. Appetite $\mathbf{6 3}$, 119-128.

43. Frisardi V, Panza F, Seripa D, et al. (2010) Nutraceutical properties of Mediterranean diet and cognitive decline: possible underlying mechanisms. J Alzheimers Dis 22, 715-740.

44. Beilharz JE, Maniam J \& Morris MJ (2015) Diet-induced cognitive deficits: the role of fat and sugar, potential mechanisms and nutritional interventions. Nutrients 7, 6719-6738.

45. Narain A, Kwok CS \& Mamas MA (2017) Soft drink intake and the risk of metabolic syndrome: a systematic review and metaanalysis. Int J Clin Pract 71, e12927.

46. Mente A, O'Donnell MJ, Rangarajan S, et al. (2014) Association of urinary sodium and potassium excretion with blood pressure. N Engl J Med 371, 601-611.

47. Nyaradi A, Li J, Hickling S, et al. (2015) A Western dietary pattern is associated with poor academic performance in Australian adolescents. Nutrients 7, 2961-2982.

48. Sörensen LB, Dyssegaard CB, Damsgaard CT, et al. (2015) The effects of Nordic school meals on concentration and school performance in 8- to 11-year-old children in the OPUS School Meal Study: a cluster-randomised, controlled, cross-over trial. Br J Nutr 113, 1280-1291.

49. Riggs NR, Spruijt-Metz D, Sakuma KL, et al. (2010) Executive cognitive function and food intake in children. $J$ Nutr Educ Behav 42, 398-403.

50. Allan JL, McMinn D \& Daly M (2016) A bidirectional relationship between executive function and health behavior: evidence, implications, and future directions. Front Neurosci 10, 386.

51. St Clair-Thompson HL \& Gathercole SE (2006) Executive functions and achievements in school: shifting, updating, inhibition, and working memory. QJ Exp Psychol (Hove) 59, 745-759.

52. Serra-Majem L, Ribas L, Ngo J, et al. (2004) Food, youth and the Mediterranean diet in Spain. Development of KIDMED, Mediterranean Diet Quality Index in children and adolescents. Public Health Nutr 7, 931-935.

53. Johnson M, Fransson G, Ostlund S, et al. (2017) Omega $3 / 6$ fatty acids for reading in children: a randomized, double-blind, placebo-controlled trial in 9-year-old mainstream schoolchildren in Sweden. J Child Psychol Psychiatry 58, 83-93. 